\title{
Analysis towards Mobile IPV4 and Mobile IPV6 in Computer Networks
}

\author{
Seyedeh Masoumeh Ahmadi \\ School of Information Technology, Guilan University, Iran \\ s_m_a57@yahoo.com
}

\begin{abstract}
With the rapid growth in the number of mobile devices like cellular phones, personal digital assistants (PDAs), and laptop computers, the demand for "anywhere, anytime, and any way" high-speed Internet access is becoming a primary concern in our lives. Mobile IP has been designed with the Internet Engineering Task Force (IETF) to serve the needs of growing population of mobile computer users who wish to connect to the internet and maintain communication as they move from place to place. Mobile IP enables a wireless network node to move freely from one point of connection to the Internet to another, without disrupting the end-to-end connectivity. The goals of this paper are to define the fundamentals of mobile IPV4, elaborate the problems of mobile IPV4, give a brief overview of some of the literature that deals with the fundamentals of mobile IPV6, explain the problems of mobile IPV6, compare Mobile IPv6 and Mobile IPv4, discuss the advantages of mobile IP, and review the application of mobile IP for vehicular networks.
\end{abstract}

Index Terms-fundamentals, mobile IPV4, mobile IPV6, advantages, application.

\section{INTRODUCTION}

With increasing popularity of mobile devices such as PDA's, internet ready cell phones, PC Tablets, etc, there is a need to provide access to the Internet that may be always in motion or wireless access to the Internet.[1] Mobile devices can be connected to the Internet by using wireless network interfaces. However, a mobile device may change its network attachment each time it moves to a new link. The necessity for uninterrupted communication when the mobile device moves from one location to another one calls for a new technology. This kind of communication can be effectively implemented using Mobile IP [2]. Mobile IP, an extension to standard Internet Protocol proposed by the Internet Engineering Task Force (IETF), aims to make mobile computing a reality and to solve the mobility problem of network node. Mobile IP refers to the mobility aspect of IP that allows nodes to move to different networks all over the world and change their point-of-attachment while maintaining all existing communications and using the same IP address. This is not to be confused with 'portability' that allows nodes to move to different networks all over the world and remain reachable, but upper-layer connections must be disrupted each time the node relocates because it has to be addressed by a new address at each location [2]. The main advantage of Mobile IP is that it frees the user from a fixed location. It makes invisible the boundaries between attachment points [3]. Mobile IP is focused on providing clear connectivity to mobile nodes in an IP based network environment while transparent mobility support is necessary for compatibility with applications [4]. Therefore, the aim of Mobile IP is to allow a mobile node to keep the same IP address in order to maintain existing connections, while remaining reachable at any new location in the Internet. It is likely the most widely known IP mobility support protocol. Two versions of 
Mobile IP have been standardized for supporting hostbased mobility on the Internet: MIPv4 and MIPv6 [5].

Mobile IPv4 is the most promising solution for mobility management in the current IPV4 network but it suffers from certain problems. Mobile IPv6 is designed for the next generation wireless networks and it overcomes the problems of MIPv4. Although MIPv6 shares many features with MIPv4, there exist some differences. The integration of mobile cell phones with Internet-based multimedia services will force a move to the next generation version of IP $(\operatorname{IPv} 6)[3]$. They support the mobility of IP hosts by allowing them to use two IP addresses: a Home Address (HoA) that shows the fixed address of a Mobile Node (MN) and a Care-ofAddress (CoA) that changes with the IP subnet to which an $\mathrm{MN}$ is currently attached. However, there are minor differences with regard to some important details. MIPv6 consists of three components: the MN, the home agent (HA), and the correspondent node $(\mathrm{CN})$. The role of the foreign agent (FA) in MIPv 4 was replaced by the access router (AR) in MIPv6. In addition, although route optimization extensions were proposed for both MIPv4 and MIPv6, they were only standardized for MIPv6 [5].

When a Mobile Node moves far away from its home network at foreign network, it will receive new address called Care-of-address (CoA). To the Correspondent Node can continue communicate to Mobile Nodes when it only knows home address of $\mathrm{MN}$, there must be a home agent in MN's home network that will forward the packets to $\mathrm{CoA}$ of $\mathrm{MN}$ [6]. The rest of this paper is organized as follows. Section 2 defines the fundamentals of mobile IPV4. Section 3 elaborates the problems of mobile IPV4. Section 4 deals with the fundamentals of mobile IPV6. Section 5 explains the problems of mobile IPV6. Section 6 compares the mobile IPV4 with mobile IPV6. Section 7 discusses the advantages of mobile IP. Section 8 reviews the application of mobile IP for vehicular networks.

\section{FUNDAMENTALS OF MOBILE IPV4}

Since IPv4 was not built with mobility in mind, Mobile IPv4 was designed as an extension to the base IPv4 protocol to support mobility. Mobile IP resolves the issue of mobility by assigning the mobile node a temporary address at each new location, maintaining the MN's original IP address, and creating and storing a covering between the two addresses with a router in the mobile node's orig inal network. [3]. MIPv 4 uses foreign agent routers functionality. MIPv4 involves a Mobile Node (MN), Home Agent (HA) that exists in home network and keeps mobility information on a $\mathrm{MN}$, and Foreign Agent (FA) that controls its network having a moving MN and acts as Care-of-Address (COA) for the $\mathrm{MN}$. And Corresponding Node $(\mathrm{CN})$ is a node that wants to connect or is communicating with the $\mathrm{MN}$ [7] Three main mechanisms of Mobile IP are agent discovering, registration, and tunnelling. The function of the three mentioned mechanis ms of Mobile IP is as follows: [8]

$>$ Agent Discovery: Mobility agents announce their availability on each link for which they provide service.

$>$ Registration: When the mobile node is away from home, it registers its care-of address with its home agent.

$>$ Tunnelling: In order for datagrams to be delivered to mobile node when it is away from home, the home agent has to tunnel the datagrams to the care-of address.

The operation of MIPv4 is shown in Figure 1.

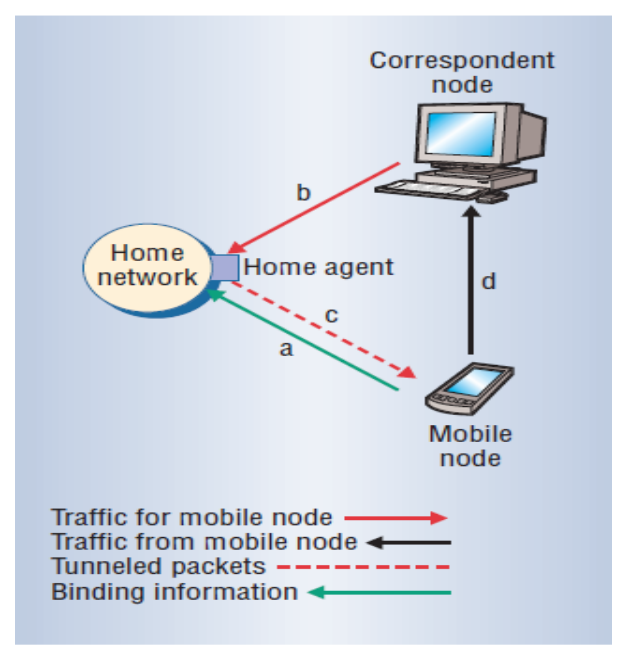

Figure1. The operation of MIPv4

As shown in Figure 1, for FA periodically sends out the agent advertisement message, $\mathrm{MN}$ receives such the 
message if $\mathrm{MN}$ enters the range controlled by FA. After receiving the message, MN starts location registration procedure by sending the registration request message with COA being FA to HA through FA. Then, HA updates mobility information on the $\mathrm{MN}$ and send the registration response and binding message. Whenever MN moves from a network to another network, HA tracks the MN's location by registration request and response. If a $\mathrm{CN}$ wants to communicate with $\mathrm{MN}$, the $\mathrm{CN}$ sends data to MN. Because of standard internet routing, data from $\mathrm{CN}$ is arrived at the home network of MN, specially HA. HA controls the binding list with the data's destination address and then transfers the data to its FA if $\mathrm{MN}$ is not in the home network. FA controls the visiting list with the destination address of the data arrived from HA by tunnelling. And then, FA directly sends the data to $\mathrm{MN}$ if $\mathrm{MN}$ is in the foreign network[3].

\section{PROBLEMS OF MOBILE IPV4}

Mobile IPv4 has many problems that need to be worked. They maybe cause communication involving Mobile IP inefficient. They have security, triangulation problems, duplication field in "IP within IP", and reliability issues. In this section, these problems are explained below.

- Security: Security is the most outstanding problem with Mobile IP. A great deal of attention is being focused on making Mobile IPv4 coexist with the security features coming into use within the internet. Firewalls, especially cause problem for Mobile IPv4 because they prevent all types of incoming packets that do not meet identified criteria. Enterprise firewalls are specifically configured to prevent packets from entering through the Internet that seem to come out from internal computers. Although this allows management of internal Internet nodes without great attention to security, it causes problems for mobile nodes within their home enterprise networks. Such communications carry the mobile node's home address and would therefore be prevented by firewall[3].
- Triangulation Problems: Triangle Routing is likely the second largest problem of Mobile IP. The main idea behind triangle routing is as follows. A mobile node wants to send a packet to another node that is on the same network. The receiver node occurs to be the far side of the Internet, far away from the mobile node's home network. Then the sending node directs all the packets to the home network. They pass through the Internet to reach the home agent and then tunnel them back across the Internet to reach the foreign agent. It would be optimal if the sending node could find out that the mobile node is on the same network and deliver the packet directly. The aim is to deliver packets as directly as possible from sending node to mobile node without passing through a home agent. This plan is called triangle routing because the single "leg" of the triangle goes from a mobile node to the corresponding node, and the home agent shapes the third vertex, controlling the path taken by the data from the correspondent node to the mobile node. This is clearly a problem since route from sender to mobile node by way of the home agent takes two sides of the triangle, rather than the third side, which is the direct path.

- Duplication field in “IP within IP": To express the datagram, we put the original datagram inside another IP envelope, then the whole packet involves the outer IP header plus the original datagram. The fields in the outer IP header add too much overhead to the final datagram several fields are copied from the inner IP header. This waste of unnecessary space is not producing profit.

- Reliability Issues: Some other issues are faced in Mobile IP. One concerns the perception of reliability, which refers to the premise of Mobile IP connections are based on TCP surviving cell changes. Many people assume that most communication systems are bursty and an increase in reliability is not needed. Another concern 
regards issues in IP addressing. Mobile IP creates a perception that a mobile node is always attached to its home network. Inefficiencies can branch from this. For example, many of communications are short lived and don't depend on the identity of the node, this does not take advantage of the idea of having a mobile node home address.

\section{FUNDAMENTALS OF MOBILE IPV6}

MIPv4 has not been used widely enough to provide much mobility and has several major shortcomings, including a communications process and a limited number of IP addresses. The latter is a key problem because the number of mobile devices that need their own IP address to access the Internet is increasing rapidly. To overcome these shortcomings and introduce new capabilities, the IETF has been developing MIPv6. MIPv6 makes many more IP addresses available and lets mobile users stay connected to the Internet as they move between networks [9]. Mobile IPv6 was designed based on the experiences gained with Mobile $\operatorname{IPv} 4$ and as such solves many of the problems identified with the previous version. The key advantage in MIPv6 is that it is based on the new IPv6 protocol. In MIPv6, IP addresses are 128 bits long integers, mobility support in MIPv 6 solves many problems of basic Mobile IP (tunnelling, ingress filter). Mobile IPv6 is in the next generation $\operatorname{IPv} 6$ network for supporting mobility [3].

There is no FA functionality in MIPv6 because MN obtained its new IPv 6 address within a foreign network by address auto-configuration method. The operational procedure of MIPv6 is shown in Figure 2.[10]

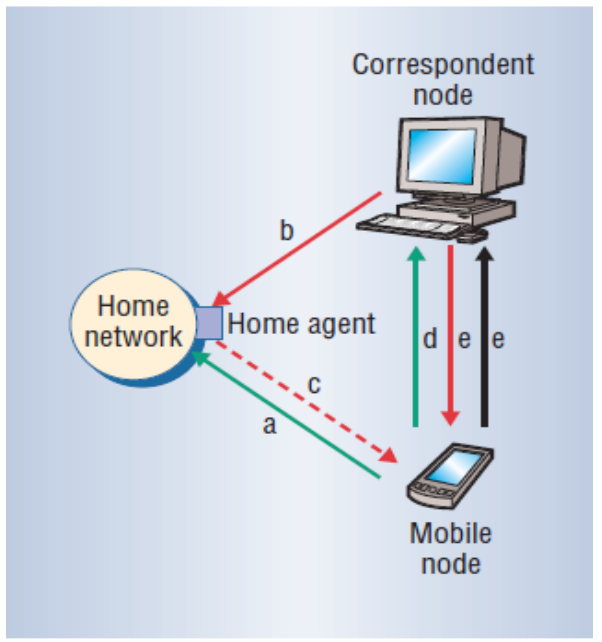

Figure 2. The operational procedure of MIPv6

In MIPv6, the MN's mobility is shown by the router advertisement message and node's neighbour unreachable detection mechanism. That is, a MN can cause a router to send its advertisement message by solicitation if necessary. After showing its mobility, the MN gets COA unlikely in MIPv4. The MN sends the binding update message to the $\mathrm{HA}$ and the communicated $\mathrm{CN}$. The $\mathrm{HA}$ and $\mathrm{CN}$ update its binding list and send their acknowledgement messages. If a $\mathrm{CN}$ wants to communicate with $\mathrm{MN}$, the $\mathrm{CN}$ sends data to MN's orig inal IP address because of not knowing MN's COA. As a result, data from $\mathrm{CN}$ is arrived at the home network through standard IP routing. The HA controls its binding list with the data's destination address and then tunnels the data to the MN's COA. The MN that tunnelled data is received send the binding update message to $\mathrm{CN}$ with understanding that the $\mathrm{CN}$ has no its own COA. After receiving the update message, the $\mathrm{CN}$ sends only data to MN's COA [10].

\section{PROBLEMS OF MOBILE IPV6}

One of the most representative efforts on the way toward next generation all-IP mobile networks is Mobile IPv6 (MIPv6) (1). Although MIPv6 is a well-known mature standard for IPv 6 mobility support and solves many problems seen in Mobile IPv4 (MIPv4) (2), it has still revealed some problems such as handover latency, packet loss, and signalling overhead. Furthermore, 
despite the reputation of this protocol, it has been slowly used in real implementations over the past years, and does not seem to receive widespread acceptance in the market [10].

\section{COMPARISON BETWEEN MOBILE IPV6 AND MOBILE IPV4}

There are two versions of Mobile IP: Mobile IP for IPv4 and IPv6. The major differences are summarised as follows [8]:

T ABLE I. COMPARISON BETWEEN MOBILE IPV6 AND MOBILE IPV4

\begin{tabular}{ccc}
\hline Key Features & $\begin{array}{c}\text { Mobile } \\
\text { IPv4 }\end{array}$ & $\begin{array}{c}\text { Mobile } \\
\text { IPv6 }\end{array}$ \\
\hline $\begin{array}{c}\text { Special router as foreign } \\
\text { agent }\end{array}$ & Yes & No \\
$\begin{array}{c}\text { Support for route } \\
\text { optimization }\end{array}$ & $\begin{array}{c}\text { Part of the } \\
\text { protocol } \\
\text { Ensure symmetric }\end{array}$ & $\begin{array}{c}\text { In } \\
\text { Extensions }\end{array}$ \\
$\begin{array}{c}\text { Noachability between mobile } \\
\text { nodes and its router at } \\
\text { current location }\end{array}$ & & Yes \\
$\begin{array}{c}\text { Routing bandwidth overhead } \\
\text { Decouple from Link Layer }\end{array}$ & More & Less \\
$\begin{array}{c}\text { Need to manage Tunnel soft } \\
\text { state }\end{array}$ & Yes & Yes \\
$\begin{array}{c}\text { Dynamic home agent } \\
\text { address discovery }\end{array}$ & No & Yes \\
\hline
\end{tabular}

\section{ADVANTAGES OF MOBILE IP}

Mobile IP is a set of standards used to adapt networking to an increasingly mobile environment. The advantages of Mobile IP are as follows:

- Convenience: Mobile IP's biggest advantage is a sustained connection to the Internet while travelling amongst a number of network connection points. In the past, a device would have to reconnect with each node as it moved along, but Mobile IP provides a simple hand-off solution that permits a device to move around seamlessly within a larger network, comprised of many connection points, without once losing their connection.

- Scalability: Since Mobile IP is built for large, expansive networks with thousands of wireless users, networks can be easily expanded to include a larger area and allow for greater connectivity for users. Whether this is on cellular networks or specific wireless LANs, a Mobile IP setup can expand to include new connection points with relative ease.

- Consistency: When a user connects to a network, they are given an IP address that identifies them on that network and permits a router connected to that portion of the network, or subnet, to send them their requested data. With Mobile IP, a user keeps the same IP address across all of the subnets they travel between while the router on their home subnet intercepts and forwards all incoming information to them across the network through a network tunnel. This provides the user with a seamless and consistent mobile connection, even if they are hosting services while moving around inside the network schema.

- Standardized: Since Mobile IP's inception, it has been adopted as a standard and is included with many advanced networking tools. Companies, such as Cisco, provide Mobile IP solutions with a number of their products, making the setup and expansion of a network using Mobile IP even easier.

- Integration: Mobile IP solutions do not need an additional network, but rather mix with a current network schema. This makes the implementation of a Mobile IP setup in any situation much easier than having to shape an additional mobile or wireless setup for interaction with the main, fixed position network.

- Uniqueness: A mobile IP address permits users to connect to the Internet without a normal static or dynamic IP address through the use of a unique mobile IP address. This unique address lets the computer connect through a network to a home IP address but still uses and communicate with the network's protocol. Having a unique IP is important for routing information to the correct computer. In short, if it were not for mobile IPs, information 
would continue to be routed to the last known IP address at which that computer was located, and a seamless connection would be impossible.

- Portability: IP creation is the only way that users can access the Internet while away from a traditional modem/router setup. Mobile IP addresses create a tunnel to a host server, which allows an access point to the Internet from any location where a signal can be received. Mobile IPs can create connection protocols to connect to the Internet through multiple servers and networks. A mobile IP address allows users to travel through multiple networks and keep an IP address. This option is useful for employees who travel throughout a building and cross into multiple wireless areas.

\section{APPLICATION OF MOBILE IP FOR VEHICULAR NETWORKS}

Vehicular ad hoc networks are gaining importance for inter-vehicle communication, because they allow for the local communication between vehicles without any infrastructure, configuration effort, and without the high costs of cellular networks [11]. Besides local data exchange, vehicular applications may be extended by accessing Internet services. In VANETs, Internet gateways (IGWs) installed at the roadside can provide a temporary Internet access, which opens up the Internet for the VANET and vice versa. This communication scenario is shown in fig. 3 where an IGW provides Internet access for the passing vehicles organized in a VANET. Vehicular applications can also consider information from the global Internet.

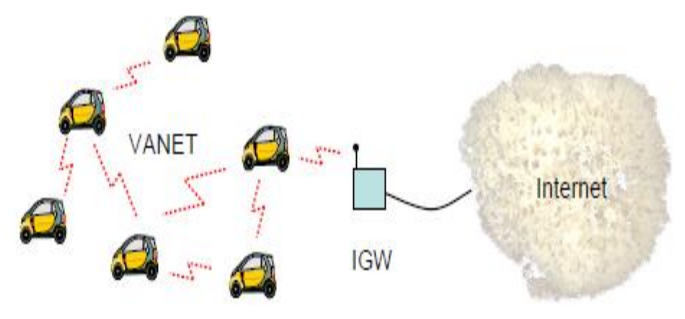

Figure 3. Internet access for the VANET
The Internet integration needs a respective mobility support of the vehicular ad hoc network. Mobile IPv 6 (RFC 3775) cannot be used for the mobility support of multihop VANETs since it always needs a direct link layer connection between gateway and mobile node [12]. For this reason, several approaches were proposed to integrate multihop ad hoc networks into the Internet using Mobile IP [13].

A mobility management protocol called MMIP6 is developed in order to control the mobility of vehicles. MMIP6 is based on the principles of Mobile IPv4, but was designed to support IPv6-based mobile nodes organized in ad hoc networks. In contrast to existing approaches, MMIP6 is optimized for scalability and efficiency. The key concept is a proactive service discovery protocol for Internet gateways providing connectivity to the Internet. This protocol is combined with an optimized mobility management protocol to control the mobility of the vehicles. Due to the proactive nature of the foreign agent discovery, MMIP6 scales well with the size of the ad hoc network. Whereas existing approaches are focused on small-scale ad hoc networking scenarios, MMIP6 is highly optimized for scalability and efficiency. Also MMIP6 is a suitable solution providing a scalable mobility support with an acceptable performance characteristic [14].

\section{CONCLUSION}

Typical mobile devices change networks several times throughout the day. When the original set of Internet protocols was designed, mobility was not an issue that was taken into account. With the rise of smart phones and increasingly more mobile devices, networking has had to adapt to accommodate the difference in how we interact with and exchange information with our network devices. Mobility support in the IP protocol has been developed by the IETF leading to the Mobile IP protocol. Mobile IP has gained attention as a technology that can provide mobility to universal users independently of the access network. Currently, two versions of Mobile IP are available, 
versions $4(\operatorname{MIPv} 4)$ and $6(\operatorname{MIPv} 6)$. Vehicular ad hoc networks become very important for inter-vehicle communication. For this reason, there is a growing need to integrate them seamlessly into the Internet, which is not possible using standard Mobile IP. The MMIP6 protocols proposed in this paper are able to integrate vehicles organized in IPv6-based multihop VANETs into the Internet.

\section{REFERENCES}

[1] C. E. Perkins, "Mobile IP," Communications Magazine, IEEE, vol. 35, pp. 84-99, 1997.

[2] C. E. Perkins, "Mobile networking through Mobile IP," Internet Computing, IEEE, vol. 2, pp. 58-69, 1998.

[3] R. Gunasundari and S. Shanmugavel, "Performance Comparison of Mobile IPv4 and Mobile IPv6 protocols in wireless systems," in Communication Systems and Networks and Workshops, 2009. COMSNETS 2009. First International, 2009, pp. 1-8.

[4] A. Yokote, A. E. Yegin, M. M. Bin Tariq, F. Guangrui, C. Williams, and A. Takeshita, "Mobile IP API," in Mobile and Wireless Communications Network, 2002. 4th International Workshop on, 2002, pp. 480-484.

[5] K. Ki-Sik, L. Wonjun, H. Youn-Hee, S. Myung-Ki, and Y. HeungRyeol, "Mobility management for all-IP mobile networks: mobile IPv6 vs. proxy mobile IPv6," Wireless Communications, IEEE, vol. 15, pp. 36-45, 2008.

[6] H. Tran Cong, C. Nguyen Ngoc, T. Nguyen Duc, and H. Truong Dinh, "Interoperability between Mobile IPv4 and Mobile IPv6 based on MPLS core network," in Advanced Communication Technology, The 9th International Conference on, 2007, pp. 1187-1190.

[7] C. Sujeong, P. Jaehyung, W. Yonggwan, Y. Mijeong, and C. Min Young, "Performance Comparison of TCP Traffic over Mobile IPv4 and IPv6 Networks and a Mobile Network Deployment Approach," in Computer and Information Technology, 2005. CIT 2005. The Fifth International Conference on, 2005, pp.469-473.

[8] X. Jiang and U. Narayanan, "Performance Analysis of Mobility Support in IPv4/IPv6 Mixed Wireless Networks," Vehicular Technology, IEEE Transactions on, vol. 59, pp. 962-973, 2010.

[9] S. J. Vaughan-Nichols, "Mobile IPv6 and the future of wireless Internet access," Computer, vol. 36, pp. 18-20, 2003.

[10] J. Younho, P. Jaehyung, W. Yonggwan, L. Bae-Ho, N. Seung Yoo, and C. Min Young, "Comparative Evaluation of TCP Performances on MIPv4 and MIPv6 Protocols in Mobile Mesh Networks," in Broadband Convergence Networks, 2007. BcN '07. 2nd IEEE/IFIP International Workshop on, 2007, pp. 1-9.
[11] W. Enkelmann, "FleetNet - applications for inter-vehicle communication," in Intelligent Vehicles Symposium, 2003. Proceedings. IEEE, 2003, pp. 162-167.

[12] S. William and M. Gerla, "IPv6 flow handoff in ad hoc wireless networks using mobility prediction," in Global Telecommunications Conference, 1999. GLOBECOM '99, 1999, pp. 271-275 vol.1a.

[13] C. Yuh-Shy an, C. Ching-Hsueh, H. Chih-Shun, and C. Ge-Ming, "Network Mobility Protocol for Vehicular Ad Hoc Networks," in Wireless Communications and Networking Conference, 2009. WCNC 2009. IEEE, 2009, pp. 1-6.

[14] M. Bechler and L. Wolf, "Mobility management for vehicular ad hoc networks," in Vehicular Technology Conference, 2005. VTC 2005-Spring. 2005 IEEE 61st, 2005, pp. 2294-2298 Vol. 4.

Seyedeh Masoumeh Ahmadi received her B.A. degree from Islamic Azad University of Lahijan, Iran. She is M.A. student in Information Technology at the Guilan University, Iran.

How to cite this paper: Seyedeh Masoumeh Ahmadi,"Analysis towards Mobile IPV4 and Mobile IPV6 in Computer Networks", International Journal of Intelligent Systems and Applications(IJISA), vol.4, no.4, pp.33-39, 2012. DOI: $10.5815 /$ ijisa.2012.04.05 